\title{
APORTES A LA CARACTERIZACIÓN Y EVALUACIÓN AGRONÓMICA DE Pourouma cecropiifolia C. Martius "Uvilla" EN LA AMAZONÍA PERUANA
}

\section{Agustín Gonzales Coral *}

\section{RESUMEN}

Una colección de árboles de Pourouma cecropiifolia C. Martius "uvilla”, del Jardín de Frutales Amazónicos del IIAP, fue caracterizada y evaluada con fines agronómicos. Las semillas fueron colectadas en el mercado Belén de Iquitos, las cuales procedían de las fincas de agricultores ubicadas en los poblados de El Dorado, 13 de Febrero, Paujil y Ex Petroleros, asentados en el eje de la carretera Iquitos - Nauta.

La evaluación incluye los aspectos de: germinación, sex ratio de plantas masculinas y femeninas, edad a la primera floración, volumen de producción a la segunda y tercera fructificación, incluyendo número de racimos por árbol, número de frutos por racimo, largo y diámetro de los frutos, pesaje de frutos, y proporciones de pulpa, semillas y cáscara.

De la evaluación de 45 plantas fueron registrados los valores promedios de frutos (2.84 cm de largo, $2.72 \mathrm{~cm}$ de ancho, $1.196 \mathrm{k} / 100$ frutos), semillas (2.24 cm de largo, $1.39 \mathrm{~cm}$ de ancho, $1.13 \mathrm{~cm}$ de espesor, $108.76 \mathrm{~g} / 100$ semillas). La masa de los frutos reporta $59 \%$ de pulpa más el mucílago, $21 \%$ semillas y $20 \%$ cáscara. La tasa de germinación de tipo hipogea es del $75 \%$, germinación que se inicia a los 25 días de sembrado y termina a los 52 días.

El rendimiento promedio en frutos es de $62292 \mathrm{~kg} /$ planta, equivalente a un rendimiento de $36799 \mathrm{~kg}$ de pulpa y mucílago. El contenido de azúcares totales varía entre 8.00 y 18.00 grados brix.

Palabras claves: Frutales nativos, Pourouma cecropiifolia, agronomía, ecología, Loreto, Amazonía Peruana.

* Instituto de Investigaciones de la Amazonía Peruana. Apto. 784.

E-mail: agonzales@iiap.org.pe Iquitos - Perú 


\begin{abstract}
A collection of trees of Pourouma cecropiifolia C. Martius "uvilla", from the Amazonian Fruit Garden of the Peruvian Amazonia Research Institute (IIAP), was characterized and evaluated with an agronomical scope. Seeds were collected at Belen market, in Iquitos. These seeds came from farms located among the towns of El Dorado, 13 de Febrero, Paujil, and Ex-Petroleros, along the Iquitos-Nauta highway.
\end{abstract}

The evaluation include germination aspects of: sex ratio of female and male plants, age of first blossom, production volume for second and third fruition including the number of clusters per tree, number of fruits per cluster, fruit length and diameter, weight, pulp portions, seeds and skins.

From the forty five (45) plants evaluated, fruit average values were registered (2.84 cm long, $2.72 \mathrm{~cm}$ wide, $1.196 \mathrm{k} / 100$ fruits), seeds ( $2.24 \mathrm{~cm}$ long, $1.39 \mathrm{~cm}$ wide, 1.13 $\mathrm{cm}$ thick, $108.76 \mathrm{~g} / 100$ seeds). Fruit mass reports $59 \%$ of pulp and mucilage, $21 \%$ seeds and $20 \%$ skin. Hipogeous germination rate is $75 \%$; it begins 25 days after sowing and ends after 52 days.

The average yield for fruits is $62292 \mathrm{k} /$ plant, equivalent to a yield of $36.799 \mathrm{k}$ of pulp and mucilage. Total sugar contents vary from 8.00 and 18.00 brix degrees.

Keywords: Native fruits, Pourouma cecropiifolia, agronomy, ecology, Loreto, Peruvian Amazonia, Peruvian Amazon

\title{
1. INTRODUCCIÓN
}

Pourouma cecropiifolia "uvilla" es un árbol frutal nativo de la Amazonía peruana, de consumo extendido en la población rural, y cuyos excedentes de producción son ofertados en los mercados. Los individuos son de comportamiento precoz, con una gran adaptación a las condiciones edafo-climáticas de la región amazónica, sin exigencias en suelo y con escasos problemas fitosanitarios, por consiguiente posee un alto potencial para la agroindustria.

En el período de 1994 a 1997, el Instituto de Investigaciones de la Amazonía Peruana (IIAP), auspició los estudios básicos de ecología, desde el manejo de las semillas hasta la cosecha de los frutos.

Las colecciones de las semillas se realizaron en el mercado de Belén, en Iquitos, donde confluyen los productos de los diferentes poblados del eje de la carretera Iquitos 
- Nauta. Las evaluaciones y el manejo de frutos y semillas comprendieron la medición, pesaje de frutos y semillas, la limpieza y desinfección de las semillas, y la germinación. La siembra de semillas se realizó en bolsas plásticas negras de $2 \mathrm{~kg}$ con substrato compuesto por materia orgánica y tierra negra. La fructificación de las plantas fue evaluada entre 3.5 y 4 años de edad.

El material vivo evaluado se conserva en el Jardín de Frutales Amazónicos, del Centro de Investigaciones Allpahuayo, del IIAP, ubicado entre los 25.8 y $28.8 \mathrm{~km}$ de la carretera Iquitos-Nauta.

\section{MATERIALES Y MÉTODOS}

\section{Materiales}

Especie, distribución geográfica, ecología

Basado en la información de Acero (1979), Calzada (1980), Brack (1987), Cavalcante (1991), Ruiz (1993), Brako \& Zarucchi (1993) y Flores (1997), se presenta la situación taxonómica y sistemática:

Familia : Cecropiaceae

Género : Pourouma

Especie $\quad:$ :Pourouma cecropiifolia C. Martius

Sinónimo : : Pourouma multifida Trecul.

Nombre común : Perú: Uvilla, Ubilla; Colombia y español: Uva caimarona, caimarón, caima; Brasil: mapati, cucura, puruma, umbauba de cheiro, imbauba de vinho, uva de mata (Brasil); Inglés: grape tree fruit.

La especie es originaria de la Amazonía Occidental, por lo que se encuentra en estado silvestre en Bolivia, Colombia, Ecuador y Perú. En Perú es cultivado por los nativos y colonos, en los departamentos de Loreto, Ucayali, San Martín, Madre de Dios, Huánuco, Amazonas, Pasco y Junín. También se cultiva en Manaos, Acre, Bahía y otros lugares adyacentes de Brasil (Prance y Silva, 1975)

Los árboles son heliófilos, dioicos, de porte mediano -5 a $10 \mathrm{~m}$ de altura-, a veces con raíces zancos, tronco recto y cilíndrico, de 20 a $40 \mathrm{~cm}$ de DAP, ramificación a partir de $\operatorname{los} 5 \mathrm{~m}$, con cicatrices en anillos entre 10-15 cm; corteza fina bien adherida, externamente cremosa, verde al rasgado que rezuma un líquido negruzco. La copa es frondosa y esférica, ramas primarias y secundarias cortas, con ramitas terminales 
horizontales u oblicuas. La madera es suave y liviana. De hojas simples, alternas, con 2 estipulas laterales caducas, pecíolo cilíndrico de 20 a $40 \mathrm{~cm}$ de largo y de 0.5 a 1.5 $\mathrm{cm}$ de diámetro; limbo coriáceo a membranáceo, haz verde oscuro brillante, envés verde plomizo glauco, lobuladas con 7 a 12 lóbulos oblonceolados de 40 por $20 \mathrm{~cm}$, ápices agudos o acuminados; palminervadas, cuyos nervios secundarios rezuman liquido negruzco al corte; pedúnculos de 10 a $15 \mathrm{~cm}$ de largo, lámina de consistencia membranácea, penninerviada.

Flores apopétalas unisexuales en diferentes plantas, inflorescencias en panículas axilares con flores unisexuales y apopétalas. Flores masculinas con 4 sépalos libres de color pardo oscuro, con 4 ó más estambres minúsculos. Flores femeninas con cáliz entero y acampanulado, ovario súpero unilocular y con un solo óvulo.

El fruto es una drupa semejante a una uva, ovoide o esférica de 2 a $4 \mathrm{~cm}$ de largo y de 1 a $4 \mathrm{~cm}$ de diámetro; epicarpio áspero, fibroso, de color verde en estado inmaduro y violáceo negro al madurar, mesocarpio blanco cristalino, pulposo, jugoso y mucilaginoso, con fibras entrecruzadas, dulce o acidulada; una sola semilla blanca semicónica a acorazonada, marrón; embrión basal con 2 por $1.5 \mathrm{~cm}$, con peso que varía entre 1.0 a $1.5 \mathrm{~g}$.

La planta presenta follaje durante todo el año, con floración entre julio y agosto y fructificación de noviembre a diciembre.

La especie se distribuye naturalmente, y se cultiva en la cuenca alta del río Amazonas, hacia las zonas limítrofes entre Colombia, Perú y Brasil, donde habitan los bosques de altura y, también, en los bosques inundables estacionalmente, conformando los "sacha manguales" Grias peruviana (Villachica, 1996).

Los rasgos ecológicos están definidos por la tolerancia de rangos de precipitación pluvial de 1,599 a $4,000 \mathrm{~mm} /$ año, temperaturas medias entre 20 y $28^{\circ} \mathrm{C}$, sin presencia de heladas.

Presenta defoliación parcial cuya intensidad y época está en función de la distribución de las lluvias. En Brasil (Jabotical, Sao Paulo), bajo condiciones subtropicales, la caída de las hojas ocurre en el invierno seco, donde además resiste las heladas suaves (Villachica, 1996).

Existe gran diversidad genética de Pourouma cecropiifolia, que se manifiesta en la arquitectura de los árboles y en las formas y tamaños de los frutos. Otras especies conocidas como «sacha uvillas», entre ellas Pourouma minor Benoist, P. bicolor C. 
Mart., P. guianesis Aublet, P. Mollis Trécul y P. tomentosa Mart. ex Miq., complementan la variedad genética.

\section{Métodos}

Las colecciones del material de propagación se realizaron mediante colecta aleatoria en el mercado Belén y fincas en el tramo de la carretera Iquitos - Nauta.

Luego de pesar y medir las semillas, se procedió a sembrar en bolsas de polietileno de color negro con substratos compuestos de $75 \%$ de materia orgánica del bosque y $25 \%$ de tierra negra. Los registros de la germinación comprendieron tiempo de inicio, tipo, tiempo de término, número de semillas germinadas, que sirvieron para estimado del porcentaje de germinación.

Las evaluaciones de precocidad de las plantas se realizaron a la primera floración, lo que permite calcular el porcentaje de plantas masculinas y femeninas. La evaluación de la producción se realiza con los conteos de racimos, pesadas, mediciones de largo y ancho de frutos, así como el nivel de sacarosa, en grados brix, de los frutos de cada planta. Los cálculos de los parámetros estadísticos se realizaron con ayuda del programa Microsoft Excel.

\section{RESULTADOS}

\section{Semillas y germinación}

Las mediciones y pesadas de las semillas, así como los parámetros de germinación de la especie son las siguientes: 
Tabla 1. Biometría de semillas y parámetros de germinación de la especie.

\begin{tabular}{|l|l|}
\hline \multicolumn{1}{|c|}{ Descriptores } & \multicolumn{1}{c|}{ Valores } \\
\hline Peso promedio de 100 semillas & $108.76 \mathrm{~g}$ \\
\hline Largo promedio de semillas & $2.24 \mathrm{~cm}$ \\
\hline Ancho promedio de semillas & $1.39 \mathrm{~cm}$ \\
\hline Espesor promedio de semillas & $1.13 \mathrm{~cm}$ \\
\hline Número de semillas sembradas & 100 \\
\hline Fecha de siembra & $27-05-94$ \\
\hline Inicio de germinación & $21-06-94$ \\
\hline Días inicio de germinación & 25 \\
\hline Término de germinación & $18-07-94$ \\
\hline Días fin de germinación & 52 \\
\hline Número de semillas germinadas & 75 \\
\hline Porcentaje de germinación & $75 \%$ \\
\hline Típo de germinación & hipogea \\
\hline
\end{tabular}

\section{Propagación}

La propagación sexual o por semillas es común, la que es adoptada como una técnica tradicional en la práctica agrícola. La semilla pierde rápidamente su viabilidad, por que no soportan tratamientos térmicos (recalcitrantes). La germinación ocurre entre los 23 a 70 días de almacigado. Cuando las semillas son puestas a germinación inmediatamente después de extraídas del fruto, la germinación generalmente es superior al $80 \%$.

El almacigado se realiza generalmente en bolsas de aproximadamente $2 \mathrm{k}$ de capacidad, conteniendo un substrato con tierra negra, arena y materia orgánica en proporción de 1:1:1. Para favorecer la emergencia de la plántula, de germinación hipógea, se recomienda que la semilla tome una posición con su sección plana en la tierra. Se colocan de 2 a 3 semillas por bolsa con humedad adecuada para asegurar una buena germinación y crecimiento de las plántulas. Luego, se procede a un entresaque, dejando de 1 a 2 plantas para el trasplante definitivo, después de 100 días de germinadas. 
Mediante la escarificación mecánica de las semillas se adelanta el inicio de germinación en 14 días; pero a los 71 días, tanto las semillas escarificadas como las que no lo están, tienen el mismo porcentaje de germinación. La reducción del contenido de humedad al 10\% disminuye totalmente el poder germinativo.

La proporción de plántulas masculinas es mayor al 50\%; de manera que es urgente el desarrollo de un método de propagación vegetativa selectiva de individuos femeninos para asegurar y garantizar una plantación y su respectiva producción agronómica.

\section{Floración y fructificación}

La primera floración, muy irregular, ha ocurrido de octubre a noviembre de 1995, a los 12 meses después del transplante; y la fructificación, en pocas plantas, entre noviembre y diciembre siguientes. La segunda floración ocurrió a los 29 meses de edad, entre los meses marzo y abril de 1997; con fructificación en los meses de mayo y junio del mismo año. La tercera floración tuvo lugar en los meses de agosto y septiembre de 1997 y consiguiente fructificación entre noviembre y diciembre. Villachica (1996) reporta que la planta empieza a fructificar a los dos años, alcanzando un óptimo de producción entre el quinto y sexto año, con disminución progresiva posterior. El mismo autor informa que la producción de racimos, en un sistema agroforestal nativo en Iquitos, es de 250/ha al segundo año, 1 000/ha en el tercero y cuarto y $5000 /$ ha al quinto año, con un peso de 1.0 a $1.8 \mathrm{k} /$ racimo; sin embargo, el periodo productivo se puede prolongar en terrenos con suelos de mayor fertilidad y con menor competencia con otras especies.

En selva baja, las experiencias indican que el período de floración corresponde entre octubre y noviembre, y la fructificación ocurre de diciembre a enero. En Manaus, la floración ocurre entre octubre y enero, con una productividad de $24.3 \mathrm{k}$ de frutos/ planta/año (Villachica, 1996). La producción media de cinco árboles en Manaus, Brasil, fue de $24.2+12.3 \mathrm{k}$ de fruta. Considerando que $26.0+4.5 \%$ esta representado por la pulpa, entonces, se tiene un promedio de $6.3 \mathrm{k}$ de pulpa aprovechable por árbol (Falçao y Lleras, 1980).

La evaluación de la producción de 45 plantas se presenta en el Tabla 2. 
Tabla 2. Evaluaciones de 45 plantas de uvilla Pourouma cecropiifolia en el Centro de Investigaciones Allpahuayo, Iquitos, Perú.

\begin{tabular}{|l|r|r|r|r|r|}
\hline Características & \multicolumn{1}{|c|}{ MAX } & PROM. & \multicolumn{1}{c|}{ MIN } & \multicolumn{1}{c|}{ D.S. } & C.V \\
\hline Altura de planta (m) & 5.95 & 4.41 & 3.00 & 0.87 & 19.73 \\
\hline $\begin{array}{l}\text { Diámetro basal de planta } \\
\text { (cm) }\end{array}$ & 15.50 & 13.29 & 8.50 & 1.55 & 12.20 \\
\hline Número de ramas & 8.00 & 6.00 & 4.00 & 1.11 & 18.50 \\
\hline Racimos por planta & 305.00 & 130.34 & 49.00 & 75.19 & 57.69 \\
\hline Frutos / racimo & 64.00 & 40.00 & 26.00 & 9.20 & 23.00 \\
\hline Frutos / planta & 12,569 & 5,134 & 1,488 & 3,004 & 58.51 \\
\hline Peso 100 frutos (g) & 1,670 & 1,196 & 0.566 & 217.00 & 19.39 \\
\hline Cáscara de 100 frutos (g) & 343 & 241 & 171 & 38.04 & 15.78 \\
\hline Semillas/100 frutos (g) & 320 & 251 & 185 & 35.12 & 13.99 \\
\hline $\begin{array}{l}\text { Pulpa + mucílago de 100 } \\
\text { frutos (g) }\end{array}$ & 1,040 & 704 & 150 & 180.06 & 25.57 \\
\hline Largo fruto (cm) & 3.19 & 2.84 & 2.28 & 0.14 & 4.93 \\
\hline Ancho de fruto (cm) & 3.09 & 2.72 & 1.93 & 0.18 & 6.62 \\
\hline Grados brix & 18.00 & 11.00 & 8.00 & 1.65 & 15.00 \\
\hline Frutos / planta (kg) & 181.728 & 62.292 & 14.594 & 41.29 & 66.60 \\
\hline $\begin{array}{l}\text { Pulpa + mucílago planta } \\
\text { (kg) }\end{array}$ & 116.238 & 36.799 & 6.362 & 26.38 & 71.30 \\
\hline Racimos por ha * & 54,290 & 23,201 & 8,722 & 13,383 & 57.68 \\
\hline $\begin{array}{l}\text { Producción de frutos } \\
\text { (TM/ha) }\end{array}$ & 32.348 & 11.088 & 2.598 & 7.35 & 66.82 \\
\hline $\begin{array}{l}\text { Peso pulpa + mucílago } \\
\text { (TM/ha) }\end{array}$ & 20.690 & 6.550 & 1.132 & 4.70 & 67.14 \\
\hline
\end{tabular}

* $\quad 178$ plantas femeninas y 22 masculinas, cantidad variable según el sistema de producción. 
Figura 1. Composición Porcentual del Fruto de Uvilla

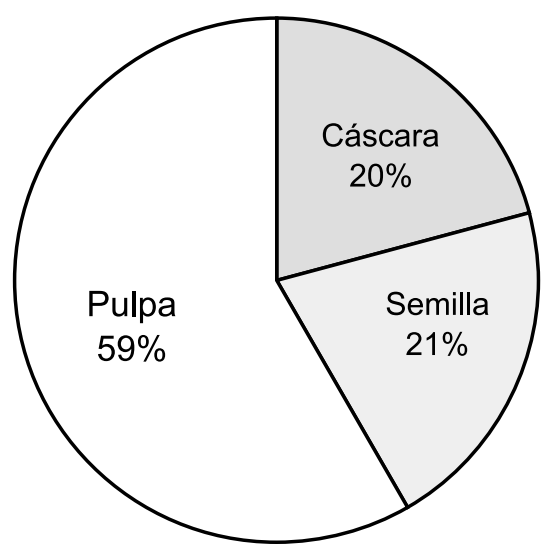

\section{Manejo del cultivo}

Los árboles de "uvilla", heliófilos y de rápido crecimiento, se comportan como componente forestal temporal de estrato medio, cuyos plantones para el transplante deben alcanzar de 50 a $60 \mathrm{~cm}$ de alto, en asociadas a otros árboles, a distancias de $6 \mathrm{~m}$, con una proporción mínima de una planta masculina por cada diez femeninas para obtener una buena producción de frutos.

Se recomienda realizar los transplantes a inicios de los períodos lluviosos, en hoyos de $25 \mathrm{~cm}$ de diámetro por $25 \mathrm{~cm}$ de profundidad, rellenados con substratos de materia orgánica y tierra de superficie. Durante los dos primeros años la práctica del deshierbo es muy importante para evitar la competencia e invasión masiva de malezas. Cuando ya están establecidos, a partir del quinto año, se recomienda el cultivo de leguminosas como cobertura.

\section{Principales plagas e insectos benéficos}

Las mayores pérdidas de los frutos son ocasionadas por los murciélagos, monos y aves frugívoras, y por pérdida de agua de los frutos durante los períodos secos muy prolongados. El "perezoso" (Bradypus tridactylus), es un mamífero devorador de hojas y yemas foliares. 
Algunos insectos de la familia Apidae (Oxytrigona obscura, Trigona dellatarreana y Trigona $s p$ ), concurren comúnmente a las flores de las "uvillas", actuando como principales agentes polinizadores entre los árboles masculinos y femeninos.

\section{Técnicas de cosechas}

Los frutos fisiológicamente maduros no se desprenden del árbol. La cosecha se practica con instrumentos rudimentarios como ganchos para jalar los racimos semimaduros (pintones). El uso de escaleras articuladas para una colecta manual facilita la colecta de frutos de mejor calidad.

Los métodos de cosechas deben mejorarse para disminuir las pérdidas de frutas por caída de los racimos.

\section{DISCUSIÓN}

En el experimento, el inicio de la germinación de las semillas fue a los 25 días, que se prolongó hasta los 52 días, con 75\%, similar a los reportes de Flores (1997) con inicio de germinación entre 23 a 25 días, y término de 42 a 51 días. Por su parte, Villachica (1996), reporta que la germinación ocurre entre los 45 y 70 días, con un adelanto de 14 días, previa escarificación; pero, a los 71 días todas las semillas tienen el mismo porcentaje de germinación. El mismo autor concluye que cuando las semillas son almacigadas, inmediatamente después de que se extraen del fruto, la germinación es generalmente superior al $80 \%$.

Los árboles evaluados alcanzaron una altura promedio de $4.41 \mathrm{~m}$, con mínimo y máximo de 3.00 y $5.95 \mathrm{~m}$, respectivamente; sin embargo, pueden tener alturas superiores entre 7 a $10 \mathrm{~m}$ de altura (Escobar \& Zuluaga s/f.); de 12 a $15 \mathrm{~m}$ (Camargo 1991); y Falçao y Lleras (1980) registraron de 2.80 a 5.50 m entre 4 y 9 años de edad. El DAP tuvo un promedio de $13.29 \mathrm{~m}$, con mínimo y máximo correspondientes a 8.50 y $15.50 \mathrm{~cm}$, pudiendo alcanzar dimensiones mayores entre 25 a $40 \mathrm{~cm}$ (Escobar y Zuluaga, s/f) y menores entre 5.10 a $8.28 \mathrm{~cm}$ (Falçao y Lleras, 1980), en Punta Negra, Manaus. El número de ramas por planta fue en promedio de 6 con sus respectivos mínimo y máximo de 4 y 8 ramas.

La primera floración de las plantas evaluadas ocurrió entre los 12 y 15 meses después de la siembra en el campo definitivo, registrándose mayor precocidad en los reportes de Yesid \& Correa (1998), Sarmiento (1986), Romero - Castañeda (1991). La 
producción de frutos en la plantación ocurrió a los 3 años de edad, un año menor al reporte de Camargo et al., (1991), y dos años menos al de La Rotta (1983), citado por Yesid \& Correa (1998). Las cosechas son estacionarias entre los meses de junio a julio, y de diciembre, enero hasta febrero, similares a los registros de Flores (1997) en Iquitos, Perú; y Hoyos (1989), en Manaus, Brasil.

El número de racimos/planta fue una característica importante, con promedio de 130, y con mínimo y máximo de 49 y 305 racimos, respectivamente; muy superiores a los registros promedios de 9.33 racimos/planta de Falçao y Lleras (1980). El número de frutos por racimos en promedio fue de 40, con mínimo de 26 y máximo de 64 frutos. Estos resultados son próximos a los de Falçao y Lleras (1980), con promedio de 56 frutos por racimo, y mínimo y máximo de 37 y 70, respectivamente. La cantidad de frutos por planta es en promedio de 62,292, con mínimo de 1,488 y máximo de 18,728; muy superiores a los reportes de Falçao y Lleras (1980), que dan un promedio de 450 y sus respectivos mínimo y máximo de 347 y 509.

La longitud promedio de los frutos fue de $2.84 \mathrm{~cm}$, con mínimo de $2.28 \mathrm{~cm}$ y máximo de $3.09 \mathrm{~cm}$, cifras próximas a los registros de Camargo (1991), cuyo promedio fue de $2.68 \mathrm{~cm}$. El diámetro promedio de los frutos fue de $2.72 \mathrm{~cm}$, con mínimo de $1.93 \mathrm{~cm}$ y máximo de $3.09 \mathrm{~cm}$; menor al promedio de $2.78 \mathrm{~cm}$, dado por Camargo (1991).

El peso de los frutos, en promedio, fue de $11.96 \mathrm{~g}$, con mínimo de $5.66 \mathrm{~g}$ y máximo de 16.70 g, mayores a los datos de Falçao y Lleras (1980), con promedio de 5.66 g, con mínimo y máximo de $5.32 \mathrm{~g}$ y $6.42 \mathrm{~g}$, respectivamente. La semilla fresca, luego de ser separada del fruto, tiene un peso promedio de $2.41 \mathrm{~g}$, con mínimo y máximo de $1.71 \mathrm{~g}$ y $3.43 \mathrm{~g}$, respectivamente; muy superiores al promedio de $1.20 \mathrm{~g}$, y mínimo de 1.01 g y máximo de 1.49 g, de Falçao y Lleras (1980).

La composición porcentual de los componentes del fruto fue 59\%, 20\% y $21 \%$ de pulpa, cáscara y semilla, respectivamente; datos que se aproximan a los de Camargo et al., (1991), con porcentaje de pulpa de $52.74 \%$. La producción de fruta y sus componentes principales (pulpa más mucílago) puede ser muy variable, dependiente de la edad de las plantas y de los sistemas de producción (monocultivos, asociaciones, etc.). En este estudio, los datos de producción obtenidos fueron de 23 mil racimos/ ha, $62 \mathrm{k}$ de frutos/árbol y $37 \mathrm{k}$ de pulpa + mucílago; superior a los reportes de Villachica (1996), quien reporta $5000 \mathrm{racimos} / \mathrm{ha}$ en un sistema agroforestal nativo en Iquitos, al cuarto año y la producción de $36.5 \mathrm{k}$ de frutos/árbol, en Manaus, Brasil, y con $6.3 \mathrm{k}$ de pulpa aprovechable por planta. 
Los contenidos de azúcares totales registrados (en grados brix), fueron en promedio de 11.00 , con mínimo y máximo de 8.00 y 18.00 , respectivamente.

\section{IMPORTANCIA PARA EL MANEJO AGROFORESTAL}

\section{Utilización y comercialización}

El mesocarpio o pulpa es la parte comestible al estado natural; también a partir de él se elaboran vinos y jaleas (Villachica, 1996). En el medio natural forma parte de la dieta de la fauna silvestre; $y$ en los centros poblados rurales los frutos se usan para la alimentación de cerdos y peces. Algunas veces se utilizan las semillas molidas como sustituto del café (Villachica 1996).

Los frutos, para fines comerciales, deben cosecharse al estado maduro en el árbol en pié, a fin de lograr óptima calidad del sabor. Sin embargo, para el comercio en los mercados alejados, los frutos deben ser cosechados en premaduración, antes de 3 a 4 días, para soportar el transporte y estibado sucesivo; sin embargo, se altera la calidad del sabor, perdiendo el sabor dulce característico. Para la industrialización, la cosecha puede estar en estado premaduro al $50 \%$, que facilita el desprendimiento de la cáscara.

En la Amazonía colombiana se utiliza la ceniza de las hojas para mezclarla con hojas de coca utilizadas para masticar («chacchar»). Del cogollo de las hojas, algunos grupos nativos extraen un líquido para aliviar las enfermedades de los ojos (Villachica, 1996).

La madera es liviana, con potencial de uso en cajonería, revestimiento de interiores, tableros aglomerados, triplay y enlistonados, entre otros.

\section{Composición química y valor nutricional}

La pulpa de los frutos en estado inmaduro tiene en promedio un $\mathrm{pH} 3.4$ y una acidez de $0.45 \%$; cuando están maduros un $\mathrm{pH} 4.4$ y una acidez de $0.16 \%$; mientras que el nivel de sacarosa, en grados brix, está en 5.5 y 11.9 para los estados respectivos. 
Tabla 3. Composición porcentual de la fruta de uvilla

\begin{tabular}{|c|c|}
\hline Componentes & $\mathbf{\%}$ \\
\hline Pulpa & 52.8 \\
\hline Mucílago & 8.8 \\
\hline Semilla & 20.6 \\
\hline Cáscara & 17.8 \\
\hline
\end{tabular}

Fuente: Villachica (1996)

Tabla 4. Análisis químico-bromatológico del fruto maduro de uvilla contenido en $100 \mathrm{~g}$ de pulpa.

\begin{tabular}{|l|l|r|}
\hline Componentes & Unidad & \multicolumn{1}{|c|}{ Valor } \\
\hline Valor energético & $\mathrm{cal}$ & 64.00 \\
\hline Agua & $\mathrm{g}$ & 82.40 \\
\hline Proteína & $\mathrm{g}$ & 0.30 \\
\hline Grasas & $\mathrm{g}$ & 0.30 \\
\hline Carbohidratos & $\mathrm{g}$ & 16.70 \\
\hline Fibras & $\mathrm{g}$ & 0.90 \\
\hline Cenizas & $\mathrm{g}$ & 0.30 \\
\hline Potasio & $\mathrm{mg}$ & 127.00 \\
\hline Calcio & $\mathrm{mg}$ & 34.00 \\
\hline Fósforo & $\mathrm{mg}$ & 10.00 \\
\hline Fierro & $\mathrm{mg}$ & 0.60 \\
\hline Riboflavina & $\mathrm{mg}$ & 0.22 \\
\hline Niacina & $\mathrm{mg}$ & 0.30 \\
\hline Ácido ascórbico & $\mathrm{mg}$ & 0.60 \\
\hline
\end{tabular}

Fuente: Villachica (1996)

\section{Aspectos agroindustriales}

El bajo contenido de azúcares y pectinas, mediana concentración de taninos y la presencia de flavonoides le confiere características promisorias en procesos de fermentación de la industria de licores, como el "vino de uvilla". En la cuenca del Putumayo, sector colombiano, se tiene una industria incipiente de "vino de caimarón". 
La pulpa al ser liberada de la cáscara toma rápidamente un color pardo, debido a la reacción de los enzimas de tipo oxidoreductasas, como las polifenoloxidasas. El tratamiento térmico a $80^{\circ} \mathrm{C}$ por $10 \mathrm{~min}$, seguido de sulfitación con 500 a 2,000 ppm de $\mathrm{SO}_{2}$, y congelación en el rango $-7 \mathrm{a}-10^{\circ} \mathrm{C}$ permite conservar el color natural de la pulpa por 30 días, aunque ocurre pérdida del aroma.

\section{RECOMENDACIONES}

\section{Necesidades de investigación}

Sobre la base de los resultados de este documento, se recomienda la urgencia de enfatizar las investigaciones en:

a. Colección y selección de variedades: Líneas que presenten los mejores rendimientos, como frutos más grandes y dulces, mayor porcentaje de pulpa, precocidad y facilidad de industrializar, entre otros.

b. Industrialización de la fruta: Para determinar las posibilidades de usos y opciones de los mercados.

c. El desarrollo de métodos de propagación vegetativa: Es muy importante, tanto para asegurar una mayor frecuencia de plantas femeninas, como para asegurar la transmisión total de las características de las plantas con germoplasma mejorado.

\section{REFERENCIAS BIBLIOGRAFICAS}

ACERO, D. E. 1979. Principales plantas útiles de la Amazonía Colombiana. Proyecto Radargamétrico del Amazonas. Bogotá pp. 135-136.

BRACK, E. W. 1987. Especies Frutales Nativas y Vegetación Melífera en la Selva Central. San Ramón. Instituto Nacional de Investigación Agraria y Agroindustrial. INIAA. p 25

BRAKO, I.; J. L. ZARUCHI. 1993. Catálogo de las Angiospermas y Gimnospermas del Perú. Missouri, EE.UU. p 343.

CALZADA, B. J. 1980. 143 Frutales Nativos. Lib. El Estudiante. Lima. Perú. 210 p. 
CAMARGO, C.; ACOSTA, M. R.; VELÁSQUEZ, M. A. 1991. Conservación de la pulpa de la uva caimarona (Pourouma cecropiaefolia). Colombia Amazónica 5(2): 27-38.

CAMARGO, C.; ACOSTA, M. R.; VELÁSQUEZ, M. A. 1991. Caracterización de la uva caimarona (Pourouma cecropiaefolia). Colombia Amazónica 5(2): 9-26.

CAVALCANTE, P. V. 1991. Frutas comestibles da Amazonia. 5a ed. Edicões CEJUP. Museo Paraense E. Goeldi, Belém. 279 p.

ESCOBAR, A. C. J.; ZULUAGA, P. J.J. (S/F), Uva caimarona (Pourouma cecropiifolia) Fruta Exótica de la Amazonia. Corpoica. Morelia Florencia Caquetá, Colombia.

FALÇAO, M. de A.; LLERAS, E. 1980. Aspectos fenológicos, ecológicos e de productividade do Mapati (Pourouma cecropiifolia Mart.). Acta amazónica $10(4): 711-724$.

FLORES, P. S. 1997. Cultivo de Frutales Nativos Amazónicos. Tratado de Cooperación Amazónica. Lima. 307 p.

PRANCE, G. T.; SILVA, M. de F. Arvores de Manaus. Manaus, AM. INPA, 1975. $312 \mathrm{p}$.

RUIZ, J. 1993. Alimentos del bosque amazónico: Una alternativa para la protección de los bosques tropicales. UNESCO/ORCYT. Montevideo. 226 p.

VILLACHICA, H. 1996. Frutales y Hortalizas Promisorias de la Amazonía. TCA. Lima. 367 p. 
ปิ)

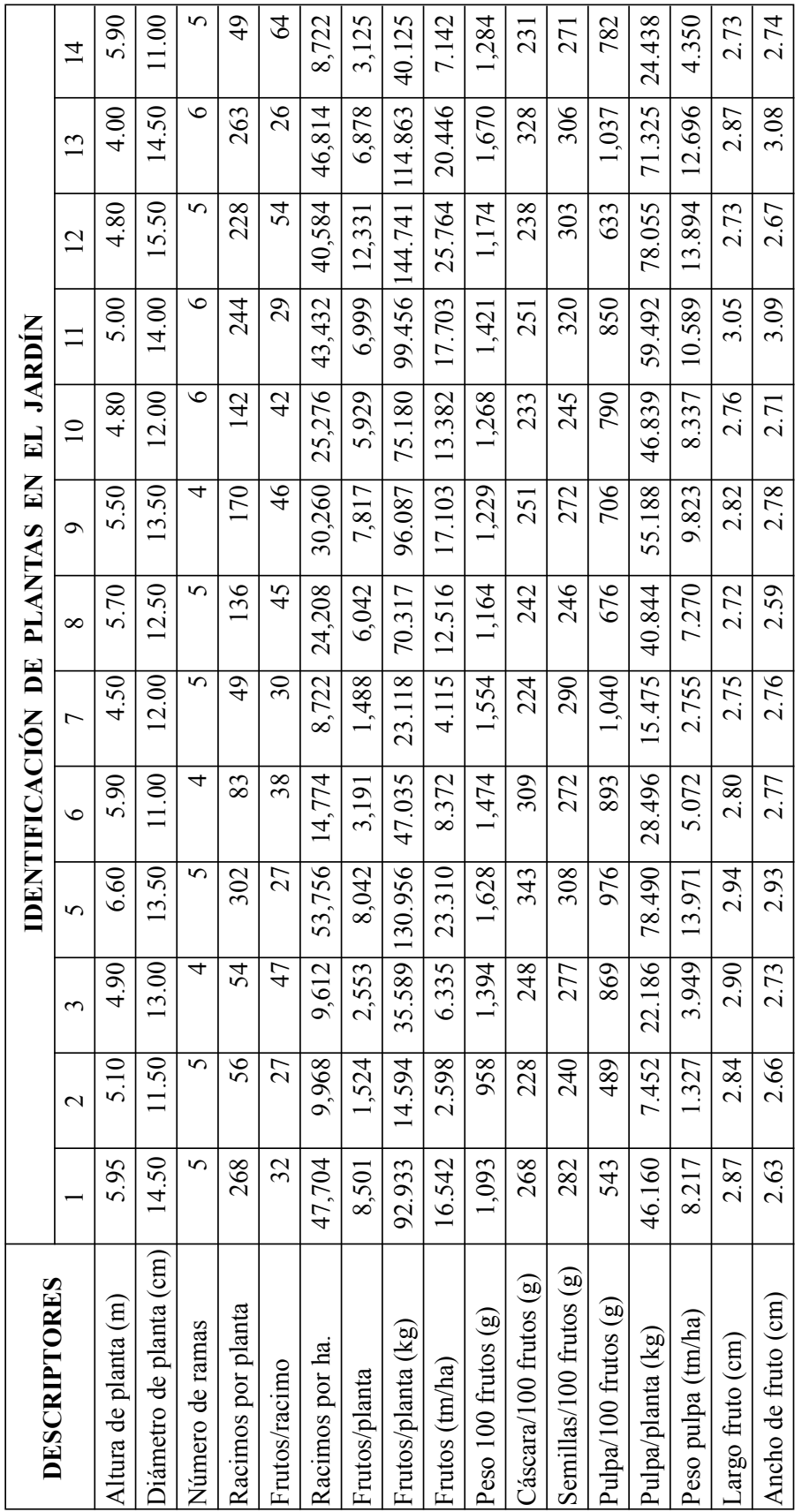




\begin{tabular}{|c|c|c|c|c|c|c|c|c|c|c|c|c|c|c|c|c|c|c|}
\hline \multirow{13}{*}{ 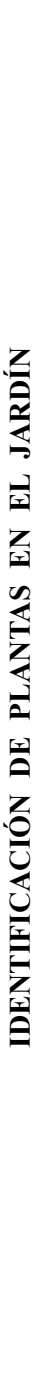 } & ㅇ & $\begin{array}{l}\stackrel{一}{\Im} \\
\text { mे }\end{array}$ & $\begin{array}{l}0 \\
n \\
\end{array}$ & $n$ & ㅇ & ले & $\begin{array}{l}\infty \\
\infty \\
0 \\
\end{array}$ & $\underset{N}{\stackrel{0}{N}}$ & $\frac{m}{\stackrel{\vartheta}{\vartheta}}$ & $\underset{\infty}{\stackrel{\infty}{r}}$ & $\begin{array}{l}m \\
\beth \\
=\end{array}$ & $\stackrel{\curvearrowright}{\curvearrowright}$ & 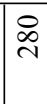 & ণิ & $\left|\begin{array}{l}\overline{0} \\
\dot{v} \\
\hat{v}\end{array}\right|$ & $\begin{array}{l}\simeq \\
\exists \\
\dot{J}\end{array}$ & ì & $\begin{array}{l}\widetilde{\sigma} \\
\stackrel{1}{0}\end{array}$ \\
\hline & Әे & $\begin{array}{l}\stackrel{8}{\Xi} \\
\dot{r}\end{array}$ & $\stackrel{\circ}{=}$ & $n$ & $\overline{6}$ & $\stackrel{\infty}{+}$ & $\begin{array}{l}\infty \\
n \\
\infty \\
0 \\
0\end{array}$ & $\begin{array}{l}\bar{n} \\
\text { a }\end{array}$ & $\begin{array}{c}a \\
\tilde{n} \\
\sim \\
\infty \\
\sim\end{array}$ & \begin{tabular}{|c|} 
\\
0 \\
0 \\
$\dot{n}$ \\
\end{tabular} & $\begin{array}{l}\infty \\
2 \\
2\end{array}$ & $\stackrel{\infty}{\underset{N}{N}}$ & 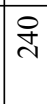 & $\stackrel{\infty}{\stackrel{\infty}{+}}$ & 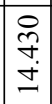 & $\begin{array}{c}3 \\
\mathfrak{o} \\
\text { i }\end{array} \mid$ & $\hat{\hat{\alpha}}$ & ñ \\
\hline & $\stackrel{\infty}{\sim}$ & $\stackrel{\circ}{n}$ & $\stackrel{\cap}{n}$ & $\nabla$ & $\stackrel{\Xi}{\Xi}$ & $\stackrel{\mathcal{Y}}{\mathcal{H}}$ & $\begin{array}{l}\text { సે } \\
\text { ภิ }\end{array}$ & 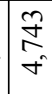 & $\begin{array}{l}\tilde{n} \\
\infty \\
\dot{b} \\
\dot{y}\end{array}$ & \begin{tabular}{|c|} 
\\
f \\
$\infty$ \\
$\infty$ \\
\end{tabular} & $\begin{array}{l}\infty \\
\infty \\
\sigma\end{array} \mid$ & $\underset{\sim}{\sim}$ & లి & $\stackrel{゚}{\stackrel{0}{+}}$ & 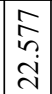 & $\begin{array}{l}a \\
0 \\
\dot{\sigma}\end{array} \mid$ & 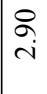 & $\stackrel{\sim}{n}$ \\
\hline & $\hat{\sim}$ & 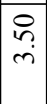 & $\begin{array}{l}i \\
n \\
n\end{array}$ & $\nabla$ & $\stackrel{\infty}{n}$ & 으 & 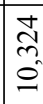 & $\begin{array}{l}\bar{\delta} \\
\text { i }\end{array}$ & $\begin{array}{l}\hat{\infty} \\
0 \\
0 \\
\dot{0}\end{array}$ & $\begin{array}{c}c \\
\delta \\
+ \\
i \\
i\end{array}$ & \begin{tabular}{l}
$\infty$ \\
\hdashline \\
\hdashline \\
-1
\end{tabular} & ๖ి & ్ㅜㅇ & ñ & $\begin{array}{l} \pm \\
0 \\
0\end{array}$ & $\begin{array}{l}0 \\
n \\
\infty \\
\sim \\
\sim\end{array} \mid$ & $\stackrel{n}{i}$ & $\stackrel{+}{\sim}$ \\
\hline & $\stackrel{n}{\sim}$ & $\begin{array}{l}\stackrel{8}{\circlearrowright} \\
+\end{array}$ & $\begin{array}{l}8 \\
\dot{\Xi} \\
\end{array}$ & $r$ & $\bar{\nabla}$ & 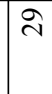 & $\begin{array}{l}\infty \\
n \\
n \\
n \\
n\end{array}$ & $\begin{array}{l}\text { तิ } \\
\text { ชీ }\end{array}$ & $\begin{array}{l}\Omega \\
\hat{\sigma} \\
\stackrel{N}{N}\end{array}$ & 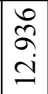 & \begin{tabular}{|l|}
$\infty$ \\
0 \\
$=$ \\
-
\end{tabular} & $\tilde{\tilde{N}}$ & $\ddot{n}$ & \& & $\begin{array}{l}\stackrel{+}{n} \\
n \\
\text { f }\end{array}$ & $\begin{array}{l}n \\
\tilde{r} \\
r\end{array}$ & ๙ু & $\stackrel{m}{i}$ \\
\hline & $\stackrel{さ}{\sim}$ & ח̊n & $\stackrel{\circ}{=}$ & 0 & 긴 & ñ & 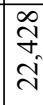 & $\begin{array}{l}\stackrel{P}{T} \\
0 \\
+\end{array}$ & $\begin{array}{l}\text { oे } \\
\text { oे } \\
\text { m. }\end{array}$ & $\begin{array}{l} \\
\\
0 \\
r \\
\end{array}$ & $\frac{m}{a}$ & $\vec{\sim}$ & $\hat{\Omega}$ & $\frac{a}{n}$ & $\begin{array}{l}\tilde{\beth} \\
\Xi \\
\end{array}$ & \begin{tabular}{|c|}
8 \\
8 \\
$r$ \\
$\dot{m}$
\end{tabular} & $\begin{array}{l}\odot \\
\infty \\
i\end{array}$ & $\begin{array}{l}\text { in } \\
\text { in }\end{array}$ \\
\hline & $\widetilde{\sim}$ & 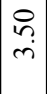 & $\begin{array}{l}\stackrel{8}{0} \\
\stackrel{\beth}{二}\end{array}$ & 0 & 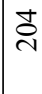 & $\bar{m}$ & 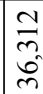 & 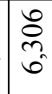 & $\begin{array}{l}\tilde{\sigma} \\
\sigma \\
\sigma\end{array}$ & $\begin{array}{l}\stackrel{P}{2} \\
\mathfrak{n} \\
\stackrel{1}{c}\end{array}$ & $\begin{array}{l}\mathfrak{Z} \\
0 \\
- \\
-\end{array}$ & $\stackrel{\infty}{\sim}$ & ฮิ & $\tilde{n}$ & 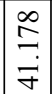 & $\begin{array}{l}0 \\
m \\
m \\
r\end{array}$ & ๙ু & 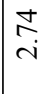 \\
\hline & $\approx$ & $\begin{array}{l}8 \\
\stackrel{+}{+}\end{array}$ & \begin{tabular}{|c|}
8 \\
\end{tabular} & $r$ & $\stackrel{ \pm}{\beth}$ & m & $\begin{array}{l}\text { त̃ } \\
0 \\
\text { ते }\end{array}$ & $\begin{array}{l}n \\
\tilde{a} \\
m\end{array}$ & $\begin{array}{l}0 \\
\infty \\
\dot{0} \\
\dot{q}\end{array}$ & 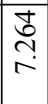 & $\begin{array}{c}\mathcal{O} \\
\mathcal{O} \\
-\end{array}$ & $\stackrel{\infty}{\varrho}$ & $\tilde{\overbrace{}}$ & $\vec{J}$ & $\begin{array}{l}2 \\
\partial \\
o \\
\end{array}$ & $\begin{array}{l}\hat{\sigma} \\
+ \\
\dot{+}\end{array}$ & $\begin{array}{l}\curvearrowright \\
\infty \\
i\end{array}$ & $\begin{array}{l}\infty \\
0 \\
\sim\end{array}$ \\
\hline & $\vec{\sim}$ & $\underset{+}{\stackrel{+}{+}}$ & $\begin{array}{l}8 \\
\dot{\Xi} \\
\end{array}$ & $r$ & $\bar{a}$ & nv & $\begin{array}{l}\stackrel{\infty}{\varrho} \\
\stackrel{-}{0}\end{array}$ & $\begin{array}{l}\text { ๖̊ } \\
\text { ๙ે }\end{array}$ & 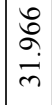 & $\begin{array}{l}8 \\
6 \\
i \mathfrak{r}\end{array}$ & $\begin{array}{l}\stackrel{8}{\circ} \\
= \\
-\end{array}$ & ৯े & $\underset{\sim}{\stackrel{J}{\sim}}$ & $\begin{array}{l}\circ \\
\stackrel{\circ}{n}\end{array}$ & $\begin{array}{l}\infty \\
\text { f } \\
\text { f. }\end{array}$ & 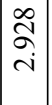 & $\frac{d}{i}$ & $\begin{array}{l}\mathbb{J} \\
\text { i }\end{array}$ \\
\hline & 2 & $\begin{array}{l}\stackrel{n}{n} \\
m\end{array}$ & $\begin{array}{l}\stackrel{\circ}{n} \\
=\end{array}$ & 6 & in & g) & $\begin{array}{l}0 \\
\text { ปे } \\
\text { సे }\end{array}$ & 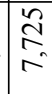 & $\begin{array}{l}\stackrel{2}{\infty} \\
\stackrel{0}{+} \\
\infty\end{array}$ & 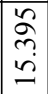 & 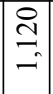 & $\underset{\sim}{\stackrel{J}{乙}}$ & $\infty$ & $\ddot{0}$ & 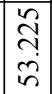 & \begin{tabular}{|c|}
\multirow{T}{*}{} \\
$\dot{\sigma}$ \\
\end{tabular} & ๙ু & $\begin{array}{l}\vec{J} \\
\stackrel{i}{ }\end{array}$ \\
\hline & $\beth$ & $\begin{array}{l}\stackrel{\circ}{n} \\
m\end{array}$ & \begin{tabular}{|l|}
8 \\
$\ddot{2}$
\end{tabular} & $n$ & in & $\underset{\forall}{\forall}$ & $\begin{array}{l}\tilde{N} \\
\tilde{O} \\
0 \\
0\end{array}$ & $\begin{array}{l}\hat{\widehat{U}} \\
\text { ป }\end{array}$ & 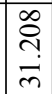 & $\begin{array}{l}n \\
n \\
n \\
n \\
\end{array}$ & $\begin{array}{l}\stackrel{\circ}{\Xi} \\
=\end{array}$ & $\stackrel{\infty}{\infty}$ & $\overline{\mathrm{d}}$ & $\underset{I}{\mathbb{I}}$ & 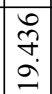 & \begin{tabular}{|c|} 
\\
8 \\
0 \\
+ \\
r.
\end{tabular} & $\begin{array}{l}\otimes \\
\infty \\
i\end{array}$ & $\begin{array}{l}\tilde{6} \\
\text { in }\end{array}$ \\
\hline & $\underline{-}$ & $\begin{array}{l}\text { ị } \\
\text { in }\end{array}$ & $\begin{array}{l} \\
n \\
n \\
\end{array}$ & $\nabla$ & $\stackrel{\Xi}{\sim}$ & $\bar{\gamma}$ & 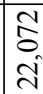 & $\underset{n}{\stackrel{O}{ \pm}}$ & $\begin{array}{l}\bar{m} \\
\dot{\infty}\end{array}$ & 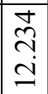 & 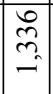 & 古 & 告 & 广্ & \begin{tabular}{|l|} 
\\
$\tilde{n}$ \\
$\tilde{y}$ \\
$\tilde{y}$
\end{tabular} & 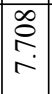 & \begin{tabular}{|l|}
$\infty$ \\
$\infty$ \\
$i$ \\
$ن$
\end{tabular} & $\begin{array}{l}\hat{\infty} \\
\text { i }\end{array}$ \\
\hline & 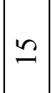 & $\begin{array}{l}\text { r. } \\
\text { in }\end{array}$ & $\begin{array}{l}8 \\
\dot{\eta}\end{array}$ & 6 & $\stackrel{n}{ \pm}$ & gे & $\begin{array}{l}0 \\
\infty \\
i n \\
\sim\end{array}$ & $\frac{\infty}{\stackrel{\infty}{ \pm}}$ & 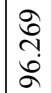 & $\begin{array}{l}0 \\
\\
\\
-\end{array}$ & $\underset{f}{\mathcal{f}}$ & లి & సి & $\stackrel{N}{N}$ & $\begin{array}{l}\stackrel{8}{0} \\
\dot{0} \\
\dot{n}\end{array}$ & $\frac{a}{a}$ & $\begin{array}{l}\dot{U} \\
\dot{m}\end{array}$ & \\
\hline & $\frac{y}{5}$ & 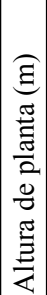 & 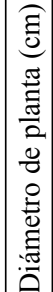 & 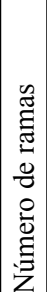 & 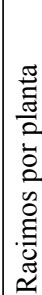 & | & 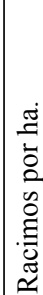 & 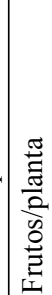 & 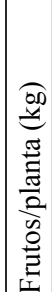 & 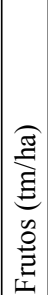 & 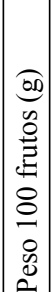 & 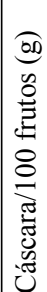 & 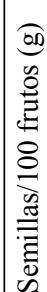 & 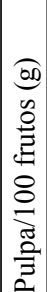 & 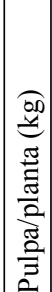 & 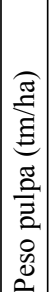 & 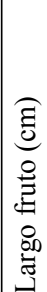 & 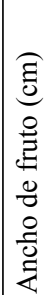 \\
\hline
\end{tabular}




\begin{tabular}{|c|c|c|c|c|c|c|c|c|c|c|c|c|c|c|c|c|c|c|}
\hline \multirow{13}{*}{ 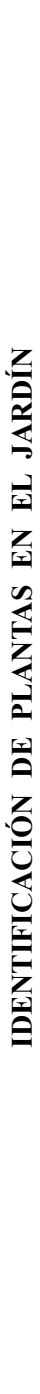 } & ষ & $\begin{array}{l}\stackrel{8}{0} \\
\dot{+}\end{array}$ & $\begin{array}{l}8 \\
\dot{\square} \\
\dot{I}\end{array}$ & $n$ & กั & in & $\begin{array}{l}\mathscr{0} \\
\infty \\
\infty \\
\dot{f}\end{array}$ & 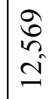 & $\begin{array}{l}a \\
0 \\
\dot{o} \\
\dot{g}\end{array}$ & 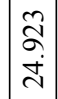 & $\begin{array}{l} \pm \\
\Xi \\
=\end{array}$ & $\infty$ & $\bar{\nabla}$ & $\frac{0}{1}$ & $\mid \begin{array}{l}\stackrel{9}{+} \\
\stackrel{2}{\infty} \\
\infty\end{array}$ & $\begin{array}{l}n \\
\infty \\
\infty \\
n \\
n\end{array}$ & ¿ & $\begin{array}{l}0 \\
0 \\
i\end{array}$ \\
\hline & $\mathscr{f}$ & $\begin{array}{l}\tilde{n} \\
\stackrel{\sim}{\forall}\end{array}$ & $\begin{array}{l}0 \\
n \\
\infty\end{array}$ & $\nabla$ & in & 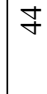 & $\begin{array}{l}\tilde{\delta} \\
\delta \\
\tilde{n} \\
0\end{array}$ & $\begin{array}{l}\hat{b} \\
\text { n } \\
\text { n. }\end{array}$ & $\vec{a}$ & $\begin{array}{c} \pm \\
n \\
n \\
n\end{array}$ & $\begin{array}{l}n \\
= \\
=\end{array}$ & $\stackrel{\circ}{-}$ & $\stackrel{\nabla}{\sim}$ & $\stackrel{N}{N}$ & $\begin{array}{l}\tilde{\approx} \\
\infty \\
\tilde{\sigma}\end{array}$ & $\begin{array}{c}\stackrel{0}{n} \\
n \\
n \\
\text { ñ }\end{array}$ & $\begin{array}{l}\text { ๙̊ } \\
\text { ণิ }\end{array}$ & $\begin{array}{l}\stackrel{8}{0} \\
\text { i }\end{array}$ \\
\hline & F & $\begin{array}{l}\stackrel{\infty}{\infty} \\
\stackrel{+}{*}\end{array}$ & $\begin{array}{l}8 \\
i \\
i\end{array}$ & $n$ & $\Xi$ & $\stackrel{\infty}{m}$ & 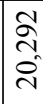 & \begin{tabular}{l}
$\infty$ \\
$\tilde{n}$ \\
\multirow{\sigma}{*}{}
\end{tabular} & $\begin{array}{l}\overrightarrow{8} \\
\dot{0} \\
\dot{\gamma}\end{array}$ & $\begin{array}{l}\text { 三 } \\
\infty\end{array}$ & $\bar{n}$ & 웟 & $\tilde{\widetilde{N}}$ & 궁 & \begin{tabular}{l}
$\mathfrak{r}$ \\
\multirow{2}{0}{} \\
$\sim$
\end{tabular} & \begin{tabular}{l}
\multirow{J}{*}{} \\
\multirow{\sigma}{*}{}
\end{tabular} & $\begin{array}{l}\tilde{\infty} \\
\infty \\
\sim\end{array}$ & $\frac{+}{i}$ \\
\hline & $\vec{\gamma}$ & $\begin{array}{l}n \\
n \\
\forall\end{array}$ & $\begin{array}{l}8 \\
i \\
i\end{array}$ & in & $\overline{\mathrm{\sigma}}$ & 우 & \begin{tabular}{l}
$\infty$ \\
$\stackrel{n}{\sigma}$ \\
\multirow{6}{0}{} \\
$\dot{t}$
\end{tabular} & $\begin{array}{l}0 \\
\infty \\
0 \\
\mathfrak{d}\end{array}$ & $\stackrel{\infty}{\stackrel{\infty}{N}}$ & 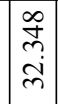 & 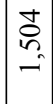 & ลิ & $\underset{\sim}{\circ}$ & రై & 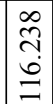 & 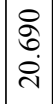 & $\begin{array}{l}\tilde{V} \\
\text { i }\end{array}$ & $\begin{array}{l}n \\
\tilde{1} \\
i\end{array}$ \\
\hline & $\stackrel{\text { \& }}{ }$ & $\begin{array}{l}\mathscr{n} \\
\stackrel{\infty}{+} \\
\end{array}$ & \begin{tabular}{|l|} 
\\
\\
$\dot{J}$
\end{tabular} & $\infty$ & 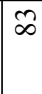 & ले & 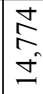 & 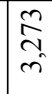 & $\begin{array}{l}\vec{n} \\
\tilde{f} \\
\text { f }\end{array}$ & $\mid \begin{array}{c}\mathbb{N} \\
\vdots \\
\infty \\
\infty\end{array}$ & 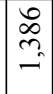 & $\stackrel{\circ}{\stackrel{0}{2}}$ & D & $\stackrel{\infty}{\infty}$ & 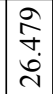 & $\underset{F}{F}$ & $\begin{array}{l}\text { ๙ั } \\
\text { ஸे }\end{array}$ & $\hat{\tilde{\gamma}}$ \\
\hline & ले & $\begin{array}{l}\tilde{m} \\
\stackrel{m}{\gamma}\end{array}$ & $\begin{array}{l}\stackrel{0}{n} \\
\dot{\Xi}\end{array}$ & $\infty$ & $\sqrt[6]{6}$ & i & $\begin{array}{l}\stackrel{8}{1} \\
\text { = } \\
=\end{array}$ & 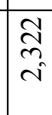 & 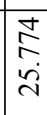 & \begin{tabular}{|l|}
$\infty$ \\
$\infty$ \\
$\wp$ \\
$\forall$ \\
\end{tabular} & $\begin{array}{l}\stackrel{0}{\varrho} \\
= \\
=\end{array}$ & 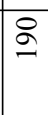 & $\widehat{\widehat{N}}$ & ลิ & $\begin{array}{l}0 \\
0 \\
0 \\
0 \\
0\end{array}$ & $\begin{array}{l}8 \\
\mathbb{0} \\
\infty \\
\text { N }\end{array}$ & $\frac{⿱}{\sim}$ & 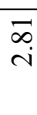 \\
\hline & $\stackrel{\infty}{\infty}$ & $\begin{array}{l}\tilde{b} \\
\dot{n}\end{array}$ & $\begin{array}{l}\stackrel{n}{n} \\
\ddot{n}\end{array}$ & $\infty$ & $\stackrel{\infty}{\circ}$ & ๙े & $\begin{array}{l}\stackrel{J}{\&} \\
\stackrel{2}{=}\end{array}$ & $\begin{array}{l}\hat{\infty} \\
\infty \\
i \\
i\end{array}$ & $\begin{array}{l}\infty \\
\infty \\
\infty \\
\infty \\
\infty\end{array}$ & $\frac{\vec{a}}{\sigma}$ & $\begin{array}{l}0 \\
\stackrel{1}{0} \\
-2 \\
-1\end{array}$ & $\stackrel{\curvearrowright}{ヘ}$ & $\underset{\sim}{\infty}$ & $\stackrel{\infty}{2}$ & \begin{tabular}{|l}
$\infty$ \\
$\tilde{n}$ \\
$\dot{v}$ \\
$\tilde{\lambda}$
\end{tabular} & \begin{tabular}{l}
$\sigma$ \\
\hdashline \\
$\dot{\sigma}$
\end{tabular} & $\begin{array}{l}\tilde{\sigma} \\
\text { v }\end{array}$ & $\begin{array}{l}\infty \\
\stackrel{v}{c}\end{array}$ \\
\hline & $\hat{m}$ & $\begin{array}{l}\stackrel{P}{m} \\
\dot{m}\end{array}$ & $\begin{array}{l}\stackrel{0}{n} \\
\dot{I}\end{array}$ & $r$ & $\overrightarrow{\mathrm{d}}$ & fo & $\begin{array}{l}\infty \\
\stackrel{2}{\infty} \\
\stackrel{f}{f}\end{array}$ & $\begin{array}{l}\tilde{O} \\
\tilde{\sigma} \\
=\end{array}$ & 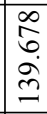 & $\mid \begin{array}{l}\hat{b} \\
\infty \\
\dot{d}\end{array}$ & $\begin{array}{l}0 \\
\stackrel{0}{0} \\
\text { רִ } \\
-\end{array}$ & స̃ & స్ & \& & $\begin{array}{l}0 \\
\mathcal{\alpha} \\
\alpha \\
\infty \\
\infty\end{array}$ & 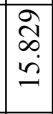 & $\overline{\widehat{\sigma}}$ & $\begin{array}{l}n \\
\infty \\
\sim\end{array}$ \\
\hline & l) & $\begin{array}{l}\curvearrowleft \\
\infty \\
\dot{f}\end{array}$ & $\begin{array}{l}8 \\
\\
\end{array}$ & in & $\bar{a}$ & $n$ & $\begin{array}{l}\infty \\
\stackrel{0}{6} \\
0\end{array}$ & $\frac{n}{n}$ & $\begin{array}{l}\infty \\
0 \\
\dot{0} \\
\dot{+}\end{array}$ & \begin{tabular}{|l|}
$\infty$ \\
$\grave{i}$ \\
$\infty$
\end{tabular} & $\begin{array}{l}\stackrel{\circ}{\mathscr{V}} \\
\stackrel{\sim}{-}\end{array}$ & $\stackrel{\sim}{\sim}$ & n & $\vec{\curvearrowleft}$ & 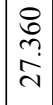 & $\begin{array}{l}\stackrel{P}{\infty} \\
\infty \\
+ \\
+\end{array}$ & 志 & $\bar{a}$ \\
\hline & $\approx$ & $\begin{array}{l}\stackrel{n}{n} \\
\text { m. }\end{array}$ & $\begin{array}{l}8 \\
\dot{m} \\
-\end{array}$ & $r$ & in & g & $\begin{array}{l}8 \\
8 \\
\infty \\
\infty\end{array}$ & $\begin{array}{l}\text { O } \\
\text { d } \\
\text { i }\end{array}$ & 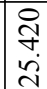 & $\begin{array}{l}\tilde{\imath} \\
\sim \\
+\end{array}$ & $\underset{\sim}{\stackrel{f}{J}}$ & $\overline{\mathrm{N}}$ & సิ & $\overrightarrow{\widetilde{\sigma}}$ & $\begin{array}{l}\tilde{n} \\
\stackrel{n}{n}\end{array}$ & $\begin{array}{l}\hat{\hat{\sigma}} \\
\text { vi }\end{array}$ & ๙ิ & $\begin{array}{l}\infty \\
\infty \\
\sim\end{array}$ \\
\hline & m & $\begin{array}{l}\mathscr{a} \\
\dot{\gamma}\end{array}$ & 号 & 0 & $\curvearrowleft$ & $n$ & $\begin{array}{l}0 \\
\hat{\sigma} \\
\hat{\sigma}\end{array}$ & $\begin{array}{l}\stackrel{8}{2} \\
\text { nn } \\
\text { n. }\end{array}$ & 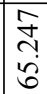 & \begin{tabular}{|l|} 
\pm \\
$\sigma$ \\
$\Xi$ \\
$=$
\end{tabular} & $\underset{\stackrel{\sim}{\sim}}{\sim}$ & $\stackrel{2}{2}$ & 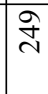 & $\infty$ & 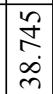 & $\begin{array}{l}\hat{a} \\
\infty \\
0\end{array}$ & $\begin{array}{l}\infty \\
\infty \\
\sim\end{array}$ & $\begin{array}{l}\circ \\
\infty \\
ن\end{array}$ \\
\hline & $\stackrel{m}{m}$ & $\begin{array}{l}\stackrel{8}{8} \\
\dot{+}\end{array}$ & $\begin{array}{l} \\
\stackrel{n}{i} \\
\text { I }\end{array}$ & $r$ & in & $\underset{\sim}{\infty}$ & $\begin{array}{l}\infty \\
0 \\
\alpha \\
\alpha\end{array}$ & $\frac{\widetilde{N}}{\sim}$ & $\begin{array}{l}\ddot{m} \\
\infty \\
\ddot{\nu}\end{array}$ & 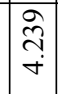 & $\begin{array}{l}\underset{I}{\cong} \\
=\end{array}$ & $\stackrel{\infty}{\curvearrowright}$ & 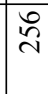 & 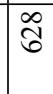 & $\begin{array}{l}\stackrel{0}{ } \\
\sim \\
\sim \\
\sim\end{array}$ & $\begin{array}{c}N \\
\tilde{N} \\
\sim\end{array}$ & $\begin{array}{l}\infty \\
\infty \\
\text { ৩ }\end{array}$ & $\begin{array}{l}+ \\
\text { i }\end{array}$ \\
\hline & m & $\begin{array}{l}\stackrel{8}{0} \\
\dot{r}\end{array}$ & 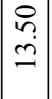 & in & $\stackrel{9}{=}$ & & $\begin{array}{l}\infty \\
\infty \\
\approx\end{array}$ & $\begin{array}{l}\frac{n}{n} \\
n \\
n\end{array}$ & $\begin{array}{l}n \\
\tilde{\infty} \\
\tilde{n} \\
\tilde{n}\end{array}$ & 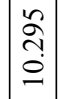 & $\begin{array}{l}0 \\
\circ \\
- \\
-1\end{array}$ & $\underset{\sim}{\approx}$ & $\underset{d}{\beth}$ & (ु) & $\begin{array}{l}\underset{J}{J} \\
\dot{m} \\
m\end{array}$ & $\begin{array}{l}n \\
\tilde{n} \\
n\end{array}$ & $\stackrel{m}{\sim}$ & $\begin{array}{l}\hat{\sigma} \\
i\end{array}$ \\
\hline & 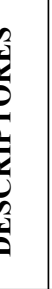 & 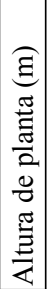 & 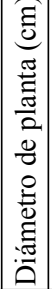 & 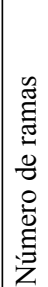 & 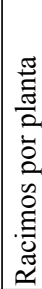 & . & 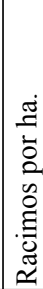 & & 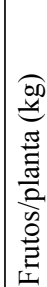 & 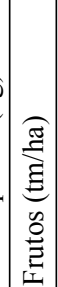 & 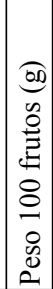 & 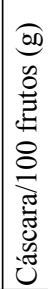 & 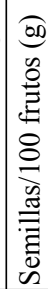 & 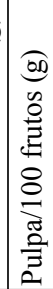 & 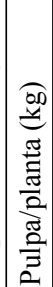 & 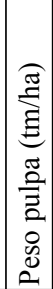 & 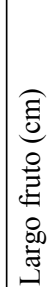 & 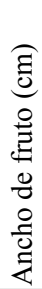 \\
\hline
\end{tabular}




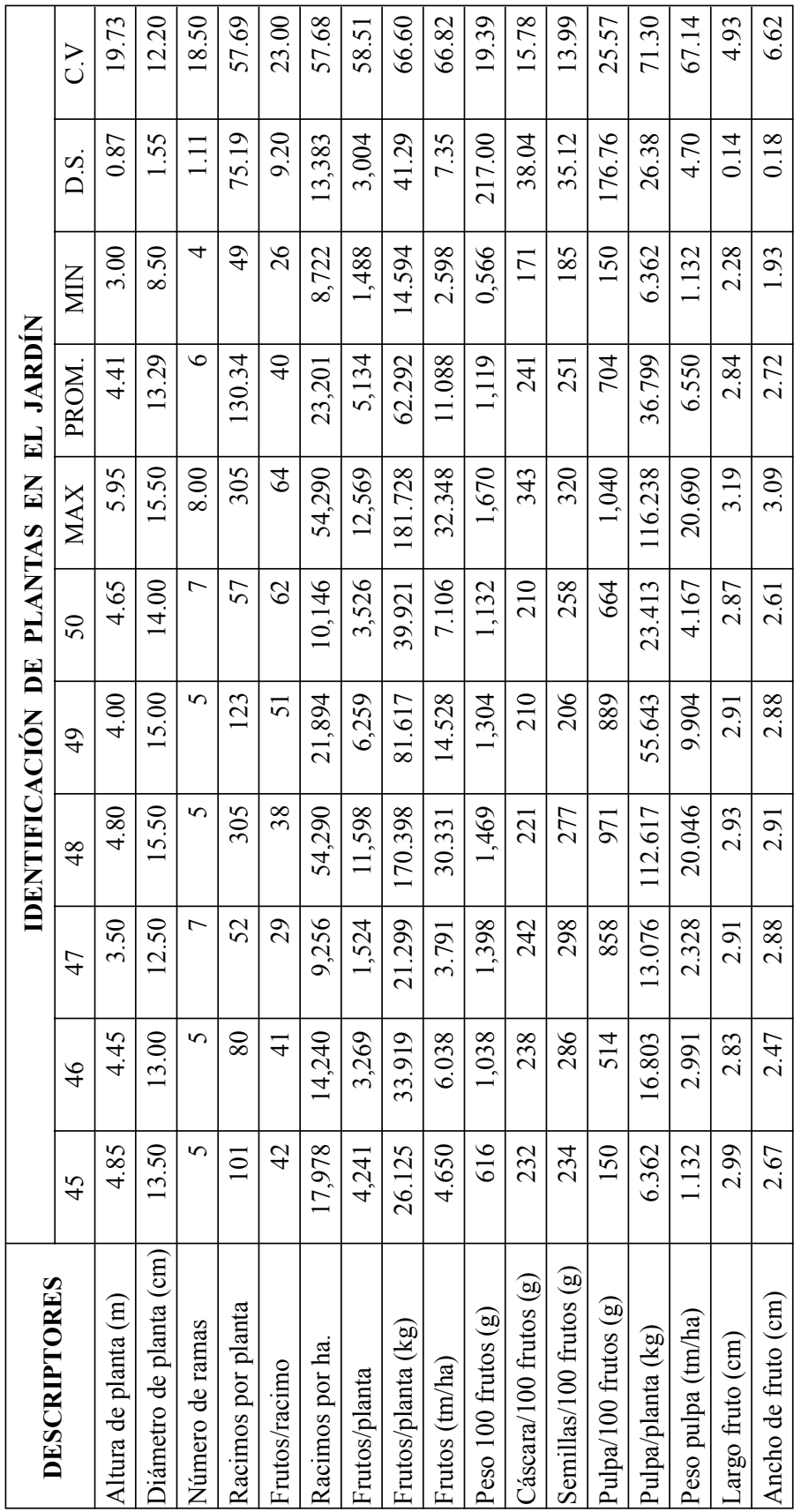

